\title{
On the source of the quasi-Carrington Rotation periodic magnetic variations on the Martian surface: InSight observations and modeling
}

\author{
Hao Luo ${ }^{1,2 *}$, AiMin Du',2, ShaoHua Zhang ${ }^{3}$, YaSong Ge ${ }^{1,2}$, Ying Zhang ${ }^{1,2}$, ShuQuan Sun ${ }^{1,2}$, Lin Zhao ${ }^{1,2}$, Lin Tian ${ }^{1,2}$, and \\ SongYan Li1,2 \\ 'Key Laboratory of Earth and Planetary Physics, Institute of Geology and Geophysics, Chinese Academy of Sciences, Beijing 100029, China; \\ ${ }^{2}$ College of Earth Science, University of Chinese Academy of Sciences, Beijing 100049, China; \\ ${ }^{3}$ Beijing Institute of Spacecraft Environment Engineering, Beijing 100094, China
}

\section{Key Points:}

- The source of the quasi-Carrington Rotation (qCR) magnetic variations is investigated by applying an analytical model.

- The ionospheric electron/plasma density is the primary factor inducing the qCR variations.

- The qCR variations could be used to probe the Martian interior's electrical conductivity structure to a depth of at least $700 \mathrm{~km}$.

Citation: Luo, H., Du, A. M., Zhang, S. H., Ge, Y. S., Zhang, Y., Sun, S. Q., Zhao, L., Tian, L., and Li, S. Y. (2022). On the source of the quasiCarrington Rotation periodic magnetic variations on the Martian surface: InSight observations and modeling. Earth Planet. Phys., 6(3), 275-283. http://doi.org/10.26464/epp2022022

\begin{abstract}
In a recent paper (Luo H et al., 2022), we found that the peak amplitudes of diurnal magnetic variations, measured during martian days (sols) at the InSight landing site, exhibited quasi Carrington-Rotation (qCR) periods at higher eigenmodes of the natural orthogonal components (NOC); these results were based on 664 sols of magnetic field measurements. However, the source of these periodic variations is still unknown. In this paper we introduce the neutral-wind driven ionospheric dynamo current model (e.g., Lillis et al., 2019) to investigate the source. Four candidates - the draped IMF, electron density/plasma density, the neutral densities, and the electron temperature in the ionosphere with artificial qCR periodicity, are applied in the modeling to find the main factor likely to be causing the observed surface magnetic field variations that exhibit the same qCR periods. Results show that the electron density/plasma density, which controls the total conductivity in the dynamo region, appears to account for the greatest part of the surface $q C R$ variations; its contribution reaches about $67.6 \%$. The draped IMF, the neutral densities, and the electron temperature account, respectively, for only about $12.9 \%, 10.3 \%$, and $9.2 \%$ of the variations. Our study implies that the qCR magnetic variations on the Martian surface are due primarily to variations of the dynamo currents caused by the electron density variations. We suggest also that the timevarying fields with the qCR period could be used to probe the Martian interior's electrical conductivity structure to a depth of at least $700 \mathrm{~km}$.
\end{abstract}

Keywords: InSight; sol magnetic variations; Carrington Rotation (CR) periodicity

\section{Introduction}

The radiation and coronal mass emitted by the Sun are not constant; they exhibit modulations at different timescales. One example is the apparent 27-day periodicity, which originates from anisotropic activities at the surface of the rotating Sun. This periodicity would be reflected in the planetary upper atmosphere, ionosphere, and dynamo current. The Interior Exploration Using Seismic Investigations, Geodesy and Heat Transport (InSight) mission, which successfully landed on Mars on 26 November 2018 at $4.50^{\circ} \mathrm{N}, 135.6^{\circ} \mathrm{E}$, has not only provided the first crustal magnetic

Correspondence to: H. Luo, luohao@mail.iggcas.ac.cn

Received 08 DEC 2021; Accepted 21 FEB 2022.

Accepted article online 11 APR 2022.

(C) 2022 by Earth and Planetary Physics. field measurements at the surface on Mars but has also monitored the ionospheric currents by measuring the time-varying magnetic field at the InSight landing site (Banfield et al., 2019; Banerdt et al., 2020; Johnson et al., 2020; Mittelholz et al., 2020). The time-varying magnetic fields offer opportunities to study solar wind-induced magnetosphere-ionosphere interactions and also to probe the electrical conductivity structure of the Martian interior (Espley et al., 2006; Johnson et al., 2020). Periodic signals, such as the daily magnetic fluctuations and their harmonics and short-period waves, were also detected by IFG (Chi PJ et al., 2019; Johnson et al., 2020). However, the solar rotation period ( 26 sols) was not detected due to the short spans of (and gaps in) the data (Johnson et al., 2020). In a recent paper we found, by organizing the magnetic field measurements for $\sim 664$ sols, that the daily amplitudes (peak-to-peak) of the magnetic variations show quasi- 
CR periodicity at higher eigenmodes of the NOC results (Luo $\mathrm{H}$ et al., 2022). However, the sources of the $q C R$ variations were not fully discussed in that paper.

Many studies have suggested that magnetic variations at the Martian surface are generated by currents in the ionospheric dynamo region (e.g., Fillingim et al., 2012; Lillis et al., 2019; Mittelholz et al., 2020). Although currents at boundaries such as the magnetic pileup boundary and bowshock boundary (Ramstad et al., 2020) may also contribute to surface magnetic variations, their contributions would be relatively small. The dynamo currents are well known to arise from differential motions of the electrons and ions in the ionospheric. In the dynamo region, the motion of the ions is dominated by neutral winds due to the higher collision frequencies. In contrast, the electrons, with much higher gyrofrequency, move mainly along the magnetic field (i.e., are magnetized). Below (above) the dynamo region, the elections and ions drift together with the neutral wind (magnetic field).

It is well known that the interplanetary magnetic field (IMF) along with the solar wind exhibit CR periodicity as the solar rotation imparts a spiral shape to the magnetic field lines (e.g. Lyons, 2003). Since Mars has no intrinsic magnetic field, the IMF could be directly draped on the Martian ionosphere. Therefore, it is expected that the CR periodicity of the magnetic field could also appear in the Martian ionosphere. Indeed, both the draped IMF intensity and direction show $\mathrm{CR}$ periodicity at the Martian induced magnetosphere/ionosphere, with synodic period between 26-27 days (e.g. Brain et al., 2006). Using Mars Global Surveyor (MGS) orbit phase observations from 2000 to 2006, Mittelholz et al. (2017) investigated the global external magnetic fields, identified 26-day periodic signals, and attributed them to the alternating polarity of the draped, compressed IMF in the 26-day CR cycle. Based also on five years of MGS measurements, Brain et al. (2006) found that the draping direction also varies on timescales associated with the solar rotation period at a given latitude band in the northern hemisphere.

The ionosphere's electron density is one of the critical parameters in inducing its dynamo current. Both temporal and spatial variations of the ionospheric irregularities (e.g., Yao MJ et al., 2019; Jiang $\mathrm{CH}$ et al., 2022) may contribute to the variation in the ionospheric dynamo current and in turn result in the magnetic field variations on the Martian surface. Therefore, periodic variations of the electron density may also result in corresponding dynamo currents with the same period. Since the Martian ionospheric peak electron density is strongly correlated with solar radiation, such as $F_{10.7}$ (e.g. Withers and Mendillo, 2005), the peak electron density at the dynamo region should also have the same solar rotation period as the solar radiation. Based on observations from the Mars Express, Nielsen et al. (2006) found that the maximum electron density at sub-solar points varies in time with the solar rotation period, which indicates contribution from ionizing solar radiation. Using MGS satellite measurements, Venkateswara Rao et al. (2014) studied statistically the electron density variation at the Martian high latitude ionosphere. They found that electron density exhibits variation with solar rotation period at nearly all altitudes. In ad- dition, the amplitude of the electron density variations was $3.5 \%-6 \%$ of the local electron density. Thus, according to Equation (1), one could expect that the dynamo currents, also, would vary with solar rotation period.

The neutral densities $\left(\mathrm{CO}_{2}, \mathrm{~N}_{2}, \mathrm{CO}\right.$, O, etc.) are believed also to have solar rotation periodic variations in Mars's thermosphere and ionosphere. In a recent paper (Hughes et al., 2022), Mars thermospheric densities (125-250 km altitude) were investigated statistically using 2015-2020 data from MAVEN's NGIMS instrument. The authors found that the 27-day periodic variation of the solar Extreme Ultra Violet (EUV) radiation has a strong impact on Mars' thermosphere densities. They also found that the effects were stronger at higher altitudes and that the relative change of the neutral densities can reach $\sim 20 \%$ at the $175 \mathrm{~km}$ altitude.

On Earth, variations in the length of the solar rotation period have long been noted in data from geomagnetic stations, e.g. Briggs (1984), who suggested that the electron density, as well as the tides wind, were sources of the $C R$ variations observed at geomagnetic stations under Solar quiet $\left(S_{q}\right)$ conditions. However, based on natural orthogonal component analysis, De Michelis et al. (2010) found that CR variations recorded at the L' Aquila observatory appeared mainly in the second principal component, which corresponds to magnetospheric currents such as the partial ring current and the field-aligned current ( $X u$ WY and Kamide, 2004). Therefore, even at Earth, sources of magnetic field variation at the surface that correlate with solar rotation period are under debate.

In this paper, based on nearly an entire martian year's worth of magnetic field measurements from InSight, we have attempted to investigate correlations between solar rotation periodicity and magnetic field variations in order to infer their possible causal relationship(s) by introducing an analytical model that imbeds the periodic ionospheric parameters to calculate ionospheric dynamo currents and corresponding magnetic field variations measured at the InSight landing site. To our best knowledge, this is the first report of and study about CR period variations of the magnetic field at the Martian surface.

\section{Data and Method}

Calibrated $0.2 \mathrm{~Hz}$ InSight IFG magnetic field data (Banfield et al., 2019) from sol 15 to sol 668 are used in this study. Figure $1 \mathrm{a}$ presents the summation of eigenmodes 2-5 of the peak-to-peak amplitude of the $H$ component in each sol, as a function of solar longitude $\left(L_{s}\right)$ and true local solar time. The thick lines in Figure 1a indicate the 10-sols running average of the peak-to-peak amplitude. We note that the amplitude varies with a quasi-CR period, with an average peak-to-peak variation amplitude of about $10 \mathrm{nT}$. Figure $1 \mathrm{~b}$ shows the corresponding FFT amplitude spectra, exhibiting quasi-CR peaks at about 27.2 days.

The model used in this study is somewhat similar to that of Lillis et al. ( 2019). According to the theory, the dynamo current can be expressed as (Lillis et al., 2019):

$$
\boldsymbol{J}_{\text {wind }}=B\left[\sigma_{p} \boldsymbol{U} \times \boldsymbol{b}+\sigma_{h} \boldsymbol{U}^{\perp}\right]
$$




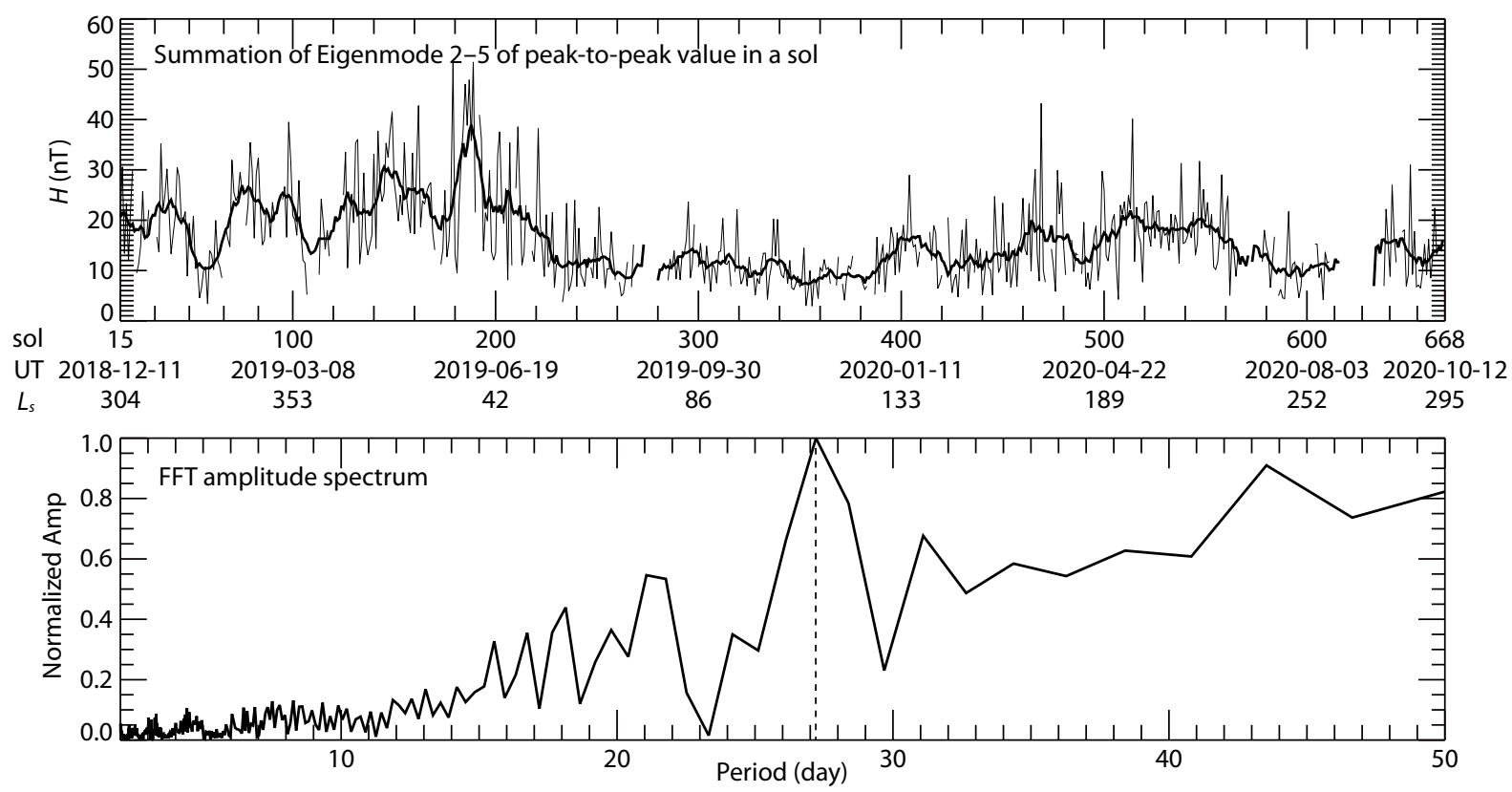

Figure 1. (a) The summation of eigenmodes $2-5$ of the peak-to-peak value of the $H$ component in a sol as a function of sols; (b) The corresponding normalized FFT amplitude spectrum. The dashed vertical line indicates the quasi-Carrington Rotation period. This figure was adapted and reconstructed from Luo $\mathrm{H}$ et al. (2022).

where $U^{\perp}$ is the neutral wind vector component perpendicular to the background magnetic field, $\boldsymbol{b}$ is the unit vector of $B, \sigma_{p}$ and $\sigma_{h}$ are the total Pedersen and Hall conductivities, and they can be written as:

$$
\begin{aligned}
& \sigma_{p}=N_{e}^{2}\left[\frac{1}{m_{i} v_{i n}\left(1+\frac{\Omega_{i}^{2}}{v_{i n}^{2}}\right)}-\left(\frac{1}{m_{e} v_{e n}\left((1+a)^{2}+\frac{\Omega_{e}^{2}}{v_{e n}^{2}}\right)}\right)\right. \\
& \left(\frac{a+\beta(1+a)}{1+\frac{\Omega_{i}^{2}}{v_{i n}^{2}}}+a\right)+\frac{N_{e}^{2}}{m_{e} v_{e n}}\left(\frac{1+a}{(1+a)^{2}+\frac{\Omega_{e}^{2}}{v_{e n}^{2}}}\right), \\
& \sigma_{h}=\frac{N_{e}^{2} \Omega_{e}}{m_{e} v_{e n}^{2}}\left(\frac{1+a}{(1+a)^{2}+\frac{\Omega_{e}^{2}}{v_{e n}^{2}}}\right)- \\
& \frac{N_{e}^{2} \Omega_{i}}{v_{i n}\left(1+\frac{\Omega_{i}^{2}}{v_{i n}^{2}}\right)}\left[\frac{1}{m_{i} v_{i n}}-\frac{a+\beta(1+a)}{m_{e} v_{e n}\left((1+a)^{2}+\frac{\Omega_{e}^{2}}{v_{e n}^{2}}\right)}\right] \text {, }
\end{aligned}
$$

where $N_{e}$ is the electron density, $\Omega_{i}=\frac{e B}{m_{i}}$ and $\Omega_{e}=\frac{e B}{m_{e}}$ are the cyclotron frequencies of the ion and electron, respectively. $a=\frac{v_{e i}}{v_{e n}}$ is a dimensionless quantity which represents the relative importance between electron-ion interactions and electron-neutral collisions. $\beta=\frac{m_{e} v_{e i}}{m_{i} v_{i n}}$ is also a dimensionless quantity reflecting the importance of electron-ion interactions to ion-neutral interactions. Other quantities, such as $v_{i n}$ and $v_{e n}$, can be determined according to Chapter 4 of Schunk and Nagy (2009). The periodicity of both the magnetic field and the conductivity could bring about periodic dynamo currents.

Here we consider several factors different from those covered in Lillis et al. ( 2019). First, we consider the dynamo currents to be varying in the horizontal plane by introducing a three-dimensional grid of the magnetic field from MAVEN observations above the InSight landing site instead of the MHD results used in Lillis et al. (2019). We have computed a statistical average of MAVEN measurements over the InSight landing site with different local times below $200 \mathrm{~km}$ altitude. Since MAVEN could not make continuous measurements above the InSight landing site at all local times, we have simply set the above statistical average value as the same for all solar longitudes $\left(L_{s}\right)$ from $0-360^{\circ}$ of InSight. Although there are seasonal variations of the magnetic field in the Martian ionosphere, we believe those variations will not influence the following modeling results in the CR time scale. Second, Ergun et al. (2015) gave the first direct in situ measurements of the electron temperature at Mars, based on data from the MAVEN Langmuir probe instrument. They investigated the altitude profile of the electron temperature and gave an analytical form:

$$
T_{e}=\frac{T_{H}+T_{L}}{2}+\frac{T_{H}-T_{L}}{2} \tanh \left(\frac{z-Z_{0}}{H_{0}}\right)
$$

where $T_{L}(0.044 \mathrm{eV} ; 510 \mathrm{~K})$ represents the asymptotic value of $T_{e}$ at the lowest altitudes, $T_{H}(0.271 \mathrm{eV} ; 3140 \mathrm{~K})$ is the high-altitude asymptotic value of $T_{e}, Z_{0}(241 \mathrm{~km})$ is the altitude of the most rapid change in $T_{e}$, and $H_{0}(60 \mathrm{~km})$ is the scale height of the rapid change. In our study, we use the electron temperature calculated according to the formula above. Finally, since the dynamo region's lower boundary greatly influences the integrated current density (Mittelholz et al., 2020), Both its upper and lower boundar- 
ies are calculated by electrons/ions gyro-frequency and electrons/ions-neutral collision(s).

To investigate which ionospheric parameter contributes the most to the solar rotation period of the surface magnetic variations observed at the InSight landing site, we artificially superpose a periodic fluctuation to the background ionospheric parameters. In addition to the magnetic field, the other background ionospheric parameters above the InSight landing site with different solar longitudes are given by the Mars Climate Database 5.3 (Millour et al., 2017), with an averaged EUV level and no dust storm scenario. This database was constructed by the Laboratoire Meteorologie Dynamiques Mars Global Circulation Model (LMD-MGCM; Forget et al., 1999; González-Galindo et al., 2013). Four categories of input parameters-the draped IMF, the electron density/ plasma density, the neutral densities, and the electron temperature with CR period-are devised to investigate which one is more important in inducing the observed periodic fluctuations at the Insight landing site. For the CR fluctuation amplitude, according to the spectral analysis of the magnetic field at 50-60 degrees of the Martian ionosphere (e.g., Mittelholz et al., 2017), the amplitude of magnetic field variations is about $10 \%$ of the local magnetic field intensity. For the electron density, both Nielsen et al.
(2006) and Venkateswara Rao et al. (2014) found evidence of a solar rotation period, and they also gave the amplitude of the fluctuation at M2 peak as approximately $3.5 \%-6 \%$ of the local electron density at Martian high latitude $\left(63^{\circ} \mathrm{N}-81^{\circ} \mathrm{N}\right)$. Here in this study we set the amplitude of the fluctuation to be $5 \%$ of the local electron density. $5 \%$ of the CR period fluctuations are also superposed to the electron temperature. $10 \%$ of the local neutral densities $\left(\mathrm{CO}_{2}\right.$, $\mathrm{O}, \mathrm{N}_{2}, \mathrm{CO}$ ) are set as the amplitude of the $\mathrm{CR}$ variations (Hughes et al., 2022). Since there is no observational evidence of the solar rotation periodicity in the neutral wind velocity in the Martian ionosphere, we do not include such fluctuations in the model.

\section{Results}

Figure 2 shows background input ionospheric parameters along with artificial fluctuations at the altitude of $130 \mathrm{~km}$, where the strongest current density is usually located (Lillis et al., 2019; Mittelholz et al., 2020). From left to right, the magnetic field, the electron density, the neutral densities, and the electron temperature, with different local true solar time and solar longitude $\left(L_{s}\right)$ are demonstrated. The magnetic field has long been known to possess a solar rotation period in both the IMF and the induced magnetosphere of Mars (e.g. Brain et al., 2006). Because neither MGS or MAVEN could make continuous measurements above the In- (a)

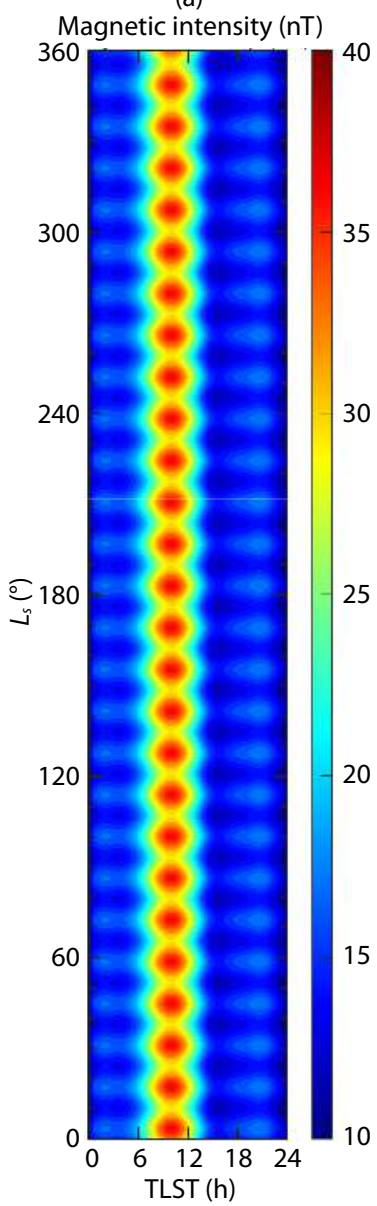

(b)

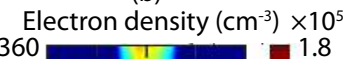

(c)

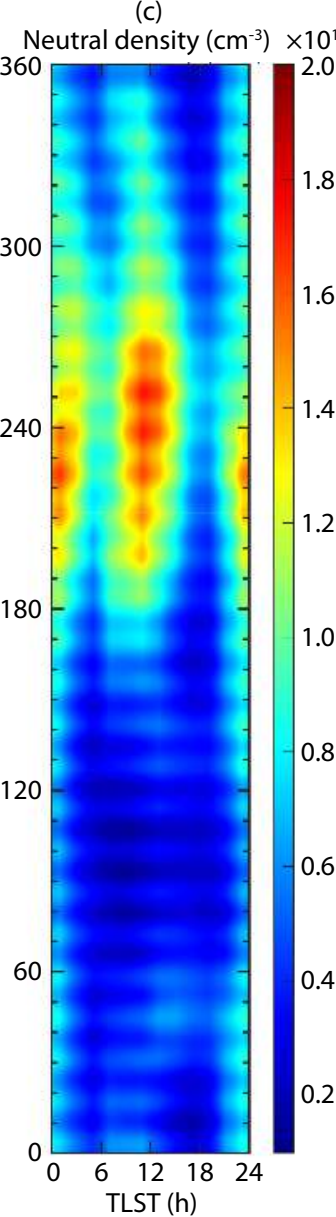

(d)

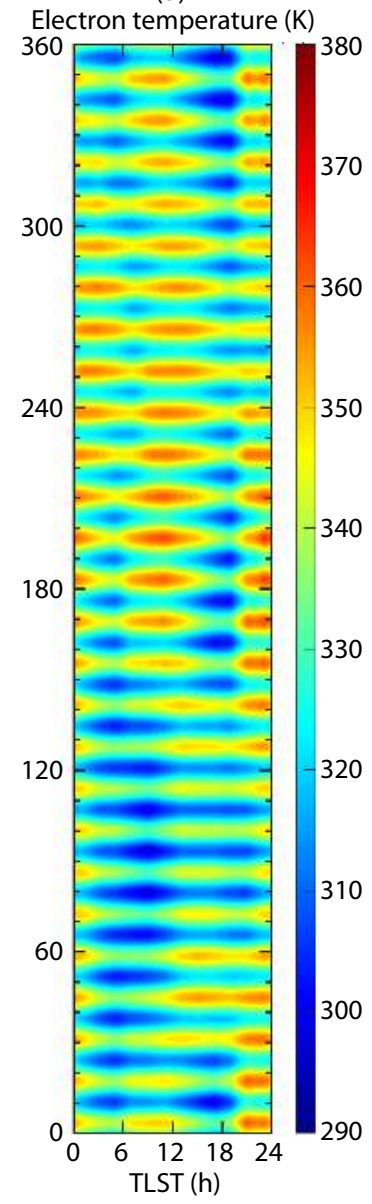

Figure 2. The model input parameters in this study at $130 \mathrm{~km}$ above the InSight landing site as a function of Ls and true local solar time: the draped IMF (a), the electron density (b), the neutral densities (c), and the electron temperature (d). 
Sight landing site with different local times and for a whole martian year, we have added periodic variations of $10 \%$ of each magnetic field component. Figure $2 a$ shows that the peak magnetic field intensity appears at dayside, indicating a pileup effect of the interplanetary magnetic field on the solar side.

As is expected, the electron density shows peaks at the subsolar point (Figure $2 \mathrm{~b}$ ). In addition, the peaks show clear seasonal dependence, with more significant electron density in perihelion, which is consistent with observations from Mars Express (Duru et al., 2019) and with MAVEN observations (Withers et al., 2015). Although there are no continuous observations above the InSight landing site, statistical studies based on data from MGS indicate that Martian high-latitude ionospheric $N_{e}$ concentrations at nearly all altitudes $(90-220 \mathrm{~km})$ reflect the solar rotation period (Venkateswara Rao et al., 2014).

The electron temperature $\left(T_{e}\right)$ is an essential parameter for electron-neutral and electron-ion collision frequency calculations (Schunk and Nagy, 2009) and is therefore critical to modeling the ionospheric dynamo at Mars (e.g., Withers et al., 2014; Cui et al., 2015; Fallows et al., 2015a, b).

The amplitude of the solar rotation periodicity of $T_{e}$, though there
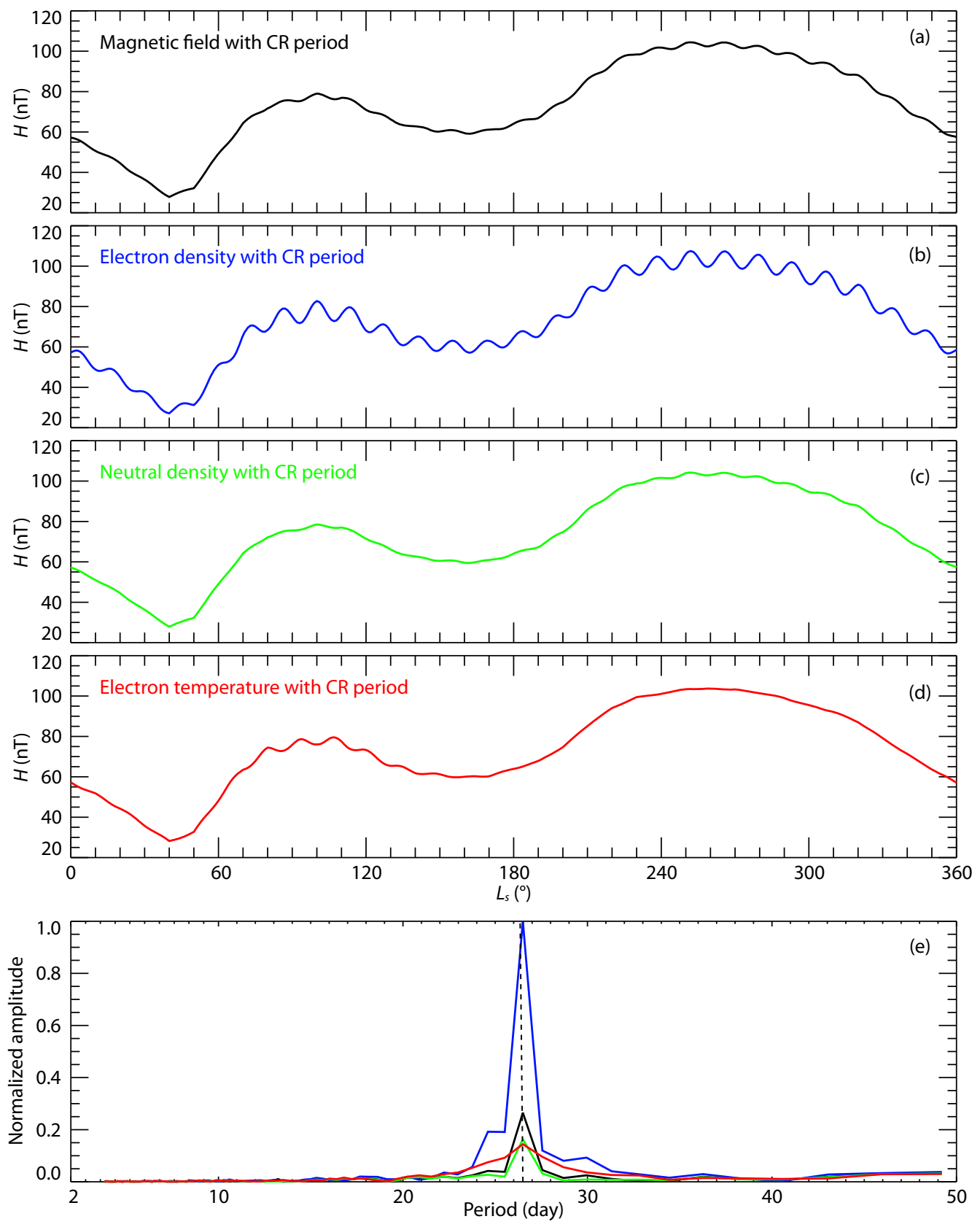

Figure 3. The simulated peak-to-peak value of the $H$ component in a sol at InSight landing site as a function of $L_{s}$ under the conditions of all the input parameters: draped IMF with CR period (a), electron density with CR period (b), neutral densities with CR period (c), and electron temperature with CR period (d); (e) the corresponding normalized amplitude spectrum. The dashed vertical line indicates the quasi-Carrington Rotation period. 
are no direct measurements, is also set to be $5 \%$ of the local $T_{e}$ over a martian year. As seen in Figure $2 \mathrm{c}$, the $T_{e}$ at $130 \mathrm{~km}$ altitude is also stronger in autumn and winter. However, the local time dependence is not as straightforward as the electron density.

Figure 3 shows simulation results of the daily amplitude (peak-topeak) of the $H$ component at the InSight landing site, with the above four input categories as a function of solar longitude $\left(L_{s}\right)$. Figures $3 a-3 d$ demonstrate the results for the periodic variations of the ionospheric draped magnetic field, the electron density, the neutral densities, and the electron temperature, respectively. In addition to their clear seasonal dependence, the fluctuations with $C R$ period are quite different for the four categories. The first category (draped IMF with CR periodicity) shows $\sim 3 \mathrm{nT}$ amplitude variation over the solar rotation period. The second category (electron density with CR periodicity) exhibits the most remarkable fluctuations with the solar rotation period; the amplitude of the electron density fluctuations is greater than $10 \mathrm{nT}$, which is comparable to the observations in Figure 1. The third category (neutral densities with CR periodicity) shows nearly the same amplitude as the first category ( 2 nT). The fourth category (electron temperature with $\mathrm{CR}$ periodicity) shows only small fluctuations near $L_{s}=120$ degree. In order to determine each category's relative quantitative contributions to the total CR periodicity of the surface magnetic field variations, we calculate the corresponding normalized FFT amplitudes for each category (Figure $3 e$ ). The FFT spectra are calculated for the interval $L_{s}$ from 0 to 360 degrees. After the quantitative calculation, the electron density/ plasma density, the IMF, the neutral densities, and electron temperature account for about $67.6 \%, 12.9 \%, 10.3 \%$, and $9.2 \%$, respectively. Results from Figures 2 and 3 indicate that the variation of the electron density in the Martian ionosphere is the main factor accounting for the CR fluctuations of the daily amplitude of the $H$ component at the Martian surface.

\section{Discussions}

\subsection{The Conductivity Variation at the Martian Ionosphere Due to Changes of Electron Density}

According to Equations (2) and (3), the Pedersen and Hall conductivities are both proportional to the plasma density (assuming that the electron density equals the ion density) in the Martian ionospheric dynamo region. Therefore, variations in the electron density at a specific position in the Martian ionosphere would induce corresponding variations of the conductivities. Figure 4 shows the total Pedersen (left panel) and Hall (right panel) conductivities at $130 \mathrm{~km}$ at different local times as a function of the $L_{s}$. The input parameters are the same as mentioned in Section 2. It was shown that the conductivities also exhibit the CR periodicity and would be the direct reason for the CR periodicity of the dynamo current and the resulting surface magnetic field. Based on measurements by the Mars Global Surveyor (MGS) during aerobraking orbits early in the mission, Opgenoorth et al. (2010) found that the most substantial layer of Pedersen conductivity is observed between 120 and $130 \mathrm{~km}$, and that Hall conductivity peaks at about $130-140 \mathrm{~km}$. The peak Pedersen and Hall conductivities
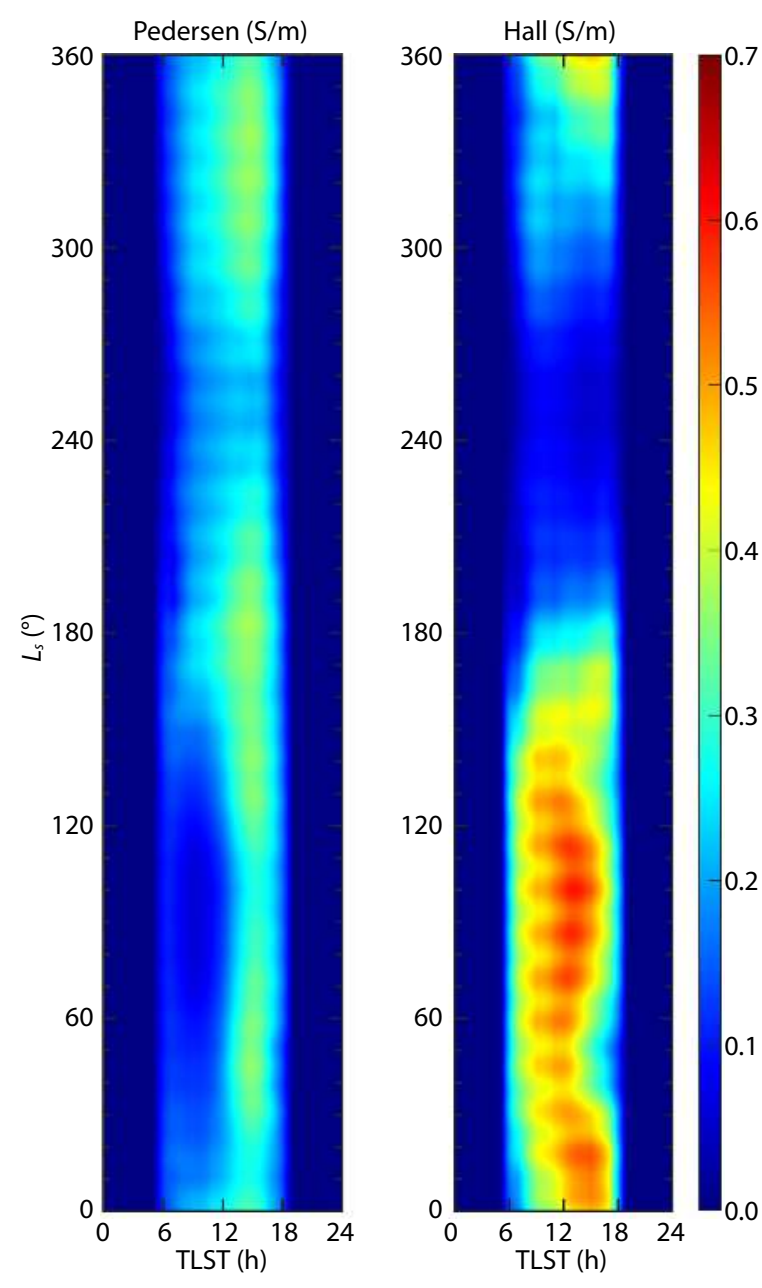

Figure 4. The Pedersen (left panel) and Hall (right panel) conductivities as a function of $L_{s}$ and true local solar time at the altitude of $130 \mathrm{~km}$ above the InSight Landing site. The electron density is the only input parameter with CR period.

can reach $0.1-1.5 \mathrm{~S} / \mathrm{m}$, which is consistent with the results in Figure 4.

\subsection{CR Effect at Martian Surface vs. at Earth's Surface}

A 27-day recurrence tendency in the Earth's ionospheric dynamo current and its corresponding daily variation at the planet's surface have been observed (e.g. Hibberd, 1981; Briggs, 1984). However, the 27-day recurrence tendency of the $S_{q}$ has appeared to be too large to be accounted for purely by changes in conductivity. Briggs (1984) believed that changes in the amplitude of the tidal winds were required to explain the observation. However, no direct evidence for such periodic variations of the tidal winds was reported in that literature. Based on the MNOC method, De Michelis et al. (2010) found that the variation in the $S_{q}$ field at the L' Aquila observatory exhibited a solar rotation period, which appeared most prominently at the second eigenmode. The second eigenmode was believed to be related to magnetospheric current systems such as the partial ring current, the related fieldaligned currents (De Michelis et al., 2010), and/or the mid-latitude return current of the substorm current system (Xu WY and Kamide, 
2004). Both the ionospheric dynamo process and the magnetospheric current system may contribute to the solar rotation periodicity observed in the magnetic field at the Earth's surface.

On the Martian surface, at the InSight landing sight, we found that the magnetic field variation with solar rotation period could be accounted for mainly by the ionospheric dynamo process. The volume current density of the Martian induced magnetospheric current, such as the induced magnetic pileup boundary (MPB) current, can reach several hundred $\mathrm{nA} / \mathrm{m}^{2}$ (Boscoboinik et al., 2020). The thickness of the MPB can vary from tens to hundreds of $\mathrm{km}$. We simply used the Biot-Savart Law to estimate the magnetic field variation at the surface, generated by an MPB of $150 \mathrm{~km}$ thickness and current density of $400 \mathrm{nA} / \mathrm{m}^{2}$. We found that the magnetic field variation generated by such an MPB current would be less than $1 \mathrm{nT}$. Nevertheless, we cannot exclude the possibility that much stronger local currents exist in the induced magnetosphere of Mars. With the successful landing of the Chinese ZhuRong Rover (Du AM et al., 2020), magnetic field measurements from two stations (InSight and ZhuRong) will be used to distinguish the magnetic effect from ionospheric dynamo current and induced magnetospheric current, since the disturbance field due to a distant induced magnetospheric current should be very similar at the two stations and could thus be canceled out, but the effects produced by an overhead ionospheric current should remain (Hibberd, 1981; Briggs, 1984; Xu, 1992; Chen et al., 2007).

The sol-to-sol daily variability in the Martian surface magnetic field is expected to be more conspicuous than is observed at the Earth's surface. One vital factor is that the Martian ionospheric dynamo process (see Equation (1)) is more dynamic than Earth's because Mars lacks a global dipole field, allowing the IMF to interact directly with the Martian ionosphere above the planet's weak crustal magnetic field (e.g., Brain et al., 2006). That is the case at the InSight landing site, above which the draped IMF and the crustal field are comparable in the dynamo region (Lillis et al., 2019; Mittelholz et al., 2020). Therefore, disturbance in the IMF could influence the total magnetic field in the dynamo region and may result in dynamo currents in the Martian ionosphere that vary far more than those in Earth's ionosphere. This expectation has been confirmed by MNOC analysis of the daily magnetic variation of InSight observations for 16-668 sols (Luo et al., 2022), in which the second eigenmode was found to be comparable with the first one. Other ionospheric parameters, such as the electron density, the neutral wind velocity, and the density, are believed to contribute less to the sol-to-sol variability than the magnetic field. However, for the CR periodicity investigated in this study, we find that electron density played a more important role than the other ionospheric parameters. Therefore, it is possible that on longer time scales, such as that of the CR period, it is the solar EUV radiation, which controls the electron density in the Martian ionosphere, that is the main influence. On the other hand, magnetic fields on shorter time scales, such as that of the diurnal and sol-tosol variability, may be attributed to the IMF variations.

It should be noted that we did not consider the CR effect of the neutral wind velocity on the daily variation on the Martian surface because no observational evidence has thus far been reported for
CR fluctuations of the neutral wind velocity in the Martian ionospheric dynamo region, though such periodicity is reported in the Earth's ionosphere (e.g., Guharay et al., 2020).

\subsection{Implication for Probing the Electric Conductivity of the Martian Interior}

One potential application of the periodic fluctuations at the Martian surface is to use this phenomenon to probe the planet's interior electric conductivity. The observed time-varying magnetic fields are the vector sum of external inducing fields and internal induced fields. The interior electric conductivity as a function of depth can be determined by separating the inducing and induced magnetic fields and analyzing the relationship between the two fields. The skin depth of time-varying magnetic fields depends on both the frequency of the inducing fields and the interior electrical conductivity structure. The Martian mantle conductivities are likely to be in the range $0.1-1 \mathrm{~S} / \mathrm{m}$ (Verhoeven and Vacher, 2016; Smrekar et al., 2019). According to estimation from Johnson et al. (2020), the magnetic fields fluctuations with CR period could be used to probe the mantle conductivities at least to a depth of $700 \mathrm{~km}$. Investigations of the upper mantle and the crust require higher frequencies, such as the daily variations and pulsation signals. Concurrent observations at different frequencies from InSight and ZhuRong magnetometers on the Mars surface, and from Tianwen-1 and MAVEN orbiting instruments, are providing unprecedented opportunities for future investigation of the interior structure of Mars.

\section{Conclusions}

In this study, by modeling the effect of several ionospheric parameters with CR periodicity on the surface magnetic field variations, we conclude as follows:

(1) Different from the Earth, the magnetic field variation with $C R$ period on the Martian surface mainly originates from the variations of the corresponding ionospheric dynamo currents.

(2) The ionospheric electron density/plasma density fluctuations related to the solar rotation contribute the most ( 68\%) to the magnetic field variation on the Martian surface with the CR period.

(3) The draped IMF, ionospheric neutral densities, and the electron temperature, which also show CR periodicity, account for the remaining $\sim 32 \%$ of the surface magnetic field variation with $C R$ periodicity.

(4) The joint observation from InSight and ZhuRong in the future is expected to be useful to distinguish the sources of the timevarying magnetic field on the Martian surface and to probe the conductivity structures in the Martian interior.

\section{Acknowledgments}

We acknowledge the Planetary Data System for access to calibrated InSight IFG data. We also acknowledge the use of the ionospheric parameters from the Mars Climate Database 5.3 (MCD 5.3). This work is supported by the Strategic Priority Research Program of the Chinese Academy of Sciences (Grant No. XDB41010304), the National Key R\&D Program of China (Grant No. 
2018YFC1503806), and the National Natural Science Foundation of China (41874080, 41674168, 41874197).

\section{References}

Banerdt, W. B., Smrekar, S. E., Banfield, D., Giardini, D., Golombek, M., Johnson, C. L., Lognonné, P., Spiga, A., Spohn, T., ... Wieczorek, M. (2020). Initial results from the InSight mission on Mars. Nat. Geosci., 13(3), 183-189. https://doi.org/10.1038/s41561-020-0544-y

Banfield, D., Rodriguez-Manfredi, J. A., Russell, C. T., Rowe, K. M., Leneman, D., Lai, H. R., Cruce, P. R., Means, J. D., Johnson, C. L., ... Banerdt, W. B. (2019). InSight Auxiliary Payload Sensor Suite (APSS). Space Sci. Rev., 215, 4. https://doi.org/10.1007/s11214-018-0570-x

Boscoboinik, G., Bertucci, C., Gomez, D., Morales, L., Mazelle, C., Halekas, J., Gruesbeck, J., Mitchell, D., Jakosky, B., and Penou, E. (2020). The magnetic structure of the subsolar MPB current layer from MAVEN observations: Implications for the Hall electric force. Geophys. Res. Lett., 47(21), e2020GL089230. https://doi.org/10.1029/2020GL089230

Brain, D. A., Mitchell, D. L., and Halekas, J. S. (2006). The magnetic field draping direction at Mars from April 1999 through August 2004. Icarus, 182(2), 464473. https://doi.org/10.1016/j.icarus.2005.09.023

Briggs, B. H. (1984). The variability of ionospheric dynamo currents. J. Atmos. Terr. Phys., 46(5), 419-429. https://doi.org/10.1016/0021-9169(84)90086-2

Chen, G. X., Xu, W. Y., Du, A. M., Wu, Y. Y., Chen, B., and Liu, X. C. (2007). Statistical characteristics of the day-to-day variability in the geomagnetic Sq field. J. Geophys. Res.: Space Phys., 112 (A6), A06320. https://doi.org/10.1029/2006JA012059

Chi, P. J., Russell, C. T., Joy, S., Banfield, D., Johnson, C. L., Ma, Y., Mittelholz, A., and $\mathrm{Yu}, \mathrm{Y}$. (2019). Magnetic pulsations on Martian surface: initial results from InSight fluxgate magnetometer. In Proceedings of the 50th Lunar and Planetary Science Conference. The Woodlands, Texas: Lunar and Planetary Institute.

Cui, J., Galand, M., Zhang, S. J., Vigren, E., and Zou, H. (2015). The electron thermal structure in the dayside Martian ionosphere implied by the MGS radio occultation data. J. Geophys. Res.: Planets, 120, 278-286. https://doi.org/10.1002/2014JE004726

De Michelis, P., Tozzi, R., and Consolini, G. (2010). Principal components' features of mid-latitude geomagnetic daily variation. Ann. Geophys., 28 , 2213-2226. https://doi.org/10.5194/angeo-28-2213-2010

Du, A. M., Zhang, Y., Li, H. Y., Qiao, D. H., Yi, Z., Zhang, T. L., Meng, L. F., Ge, Y. S., Luo, H., ... Dai, J. L. (2020). The Chinese Mars ROVER fluxgate magnetometers. Space Sci. Rev., 216(8), 135. https://doi.org/10.1007/s11214-020-00766-8

Duru, F., Brain, B., Gurnett, D. A., Halekas, J., Morgan, D. D., and Wilkinson, C. J. (2019). Electron density profiles in the upper ionosphere of Mars from 11 years of MARSIS data: Variability due to seasons, solar cycle, and crustal magnetic fields. J. Geophys. Res.: Space Phys., 124(4), 3057-3066. https://doi.org/10.1029/2018JA026327

Ergun, R. E., Morooka, M. W., Andersson, L. A., Fowler, C. M., Delory, G. T., Andrews, D. J., Eriksson, A. I., McEnulty, T., and Jakosky, B. M. (2015). Dayside electron temperature and density profiles at Mars: First results from the MAVEN Langmuir probe and waves instrument. Geophys. Res. Lett., 42(21), 8846-8853. https://doi.org/10.1002/2015GL065280

Espley, J. R., Delory, G. T., and Cloutier, P. A. (2006). Initial observations of lowfrequency magnetic fluctuations in the Martian ionosphere. J. Geophys. Res.: Planets, 111(E6), E06S22. https://doi.org/10.1029/2005JE002587

Fallows, K., Withers, P., and Matta, M. (2015a). An observational study of the influence of solar zenith angle on properties of the M1 layer of the Mars ionosphere. J. Geophys. Res.: Space Phys., 120(2), 1299-1310. https://doi.org/10.1002/2014JA020750

Fallows, K., Withers, P., and Matta, M. (2015b). Numerical simulations of the influence of solar zenith angle on properties of the M1 layer of the Mars ionosphere. J. Geophys. Res.: Space Phys., 120(8), 6707-6721.
https://doi.org/10.1002/2014JA020947

Fillingim, M. O., Lillis, R. J., England, S. L., Peticolas, L. M., Brain, D. A., Halekas, J. S., Paty, C., Lummerzheim, D., and Bougher, S. W. (2012). On wind-driven electrojets at magnetic cusps in the nightside ionosphere of Mars. Earth Planets Space, 64(2), 5. https://doi.org/10.5047/eps.2011.04.010

Forget, F., Hourdin, F., Fournier, R., Hourdin, C., Talagrand, O., Collins, M., Lewis, S. R., Read, P. L., and Huot, J. P. (1999). Improved general circulation models of the Martian atmosphere from the surface to above $80 \mathrm{~km}$. J. Geophys. Res.: Planets, 104(E10), 24155-24175. https://doi.org/10.1029/1999JE001025

González-Galindo, F., Chaufray, J. Y., López-Valverde, M. A., Gilli, G., Forget, F., Leblanc, F., Modolo, R., Hess, S., and Yagi, M. (2013). Three-dimensional Martian ionosphere model: I The photochemical ionosphere below $180 \mathrm{~km}$. J. Geophys. Res.: Planets, 118(10), 2105-2123. https://doi.org/10.1002/Jgre.20150

Guharay, A., Batista, P. P., Buriti, R. A., and Schuch, N. J. (2020). Signature of the 27-day oscillation in the MLT tides and its relation with solar radiation at low latitudes. Earth Planets Space, 72, 51. https://doi.org/10.1186/s40623020-01149-7

Hibberd, F. H. (1981). Day-to-day variability of the Sq geomagnetic field variation. Aust. J. Phys., 34(1), 81-90. https://doi.org/10.1071/PH810081

Hughes, J., Gasperini, F., and Forbes, J. M. (2022). Solar rotation effects in Martian thermospheric density as revealed by five years of MAVEN observations. J. Geophys. Res.: Planets, 127, e2021 JE007036. https://doi.org/10.1029/2021JE007036

Jiang, C. H., Tian, R., Wei, L. H., Yang, G. B., and Zhao, Z. Y. (2022). Modeling of kilometer-scale ionospheric irregularities at Mars. Earth Planet. Phys., 6(2), 213-217. https://doi.org/10.26464/epp2022011

Johnson, C. L., Mittelholz, A., Langlais, B., Russell, C. T., Ansan, V., Banfield, D., Chi, P. J., Fillingim, M. O., Forget, F., ... Banerdt, W. B. (2020). Crustal and time-varying magnetic fields at the InSight landing site on Mars. Nat. Geosci., 13(3), 199-204. https://doi.org/10.1038/s41561-020-0537-x

Lillis, R. J., Fillingim, M. O., Ma, Y. J., Gonzalez-Galindo, F., Forget, F., Johnson, C. L., Mittelholz, A., Russell, C. T., Andersson, L., and Fowler, C. M. (2019). Modeling wind-driven ionospheric dynamo currents at Mars: Expectations for InSight magnetic field measurements. Geophys. Res. Lett., 46(10), 5083-5091. https://doi.org/10.1029/2019GL082536

Luo, H., Du, A. M., Ge, Y. S., Johnson, C. L., Mittelholz, A., Zhang, Y., Sun, S. Q., Zhao, L., Yu, Y., ... Xu, W. Y. (2022). Natural orthogonal component analysis of daily magnetic variations at the Martian surface: InSight observations. J. Geophys. Res.: Planets, 127(2), e2021JE007112. https://doi.org/10.1029/2021JE007112

Lyons, L. (2003). Space Plasma Physics in Encyclopedia of Physical Science and Technology (3rd ed).

Millour, E., Forget, F., and Lewis, S. R. (2017), The Mars Climate Database (MCD version 5.3), 2017/04/1.

Mittelholz, A., Johnson, C. L., and Lillis, R. J. (2017). Global-scale external magnetic fields at Mars measured at satellite altitude. J. Geophys. Res.: Planets, 122(6), 1243-1257. https://doi.org/10.1002/2017JE005308

Mittelholz, A., Johnson, C. L., Thorne, S. N., Joy, S., Barrett, E., Fillingim, M. O., Forget, F., Langlais, B., Russell, C. T., ... Banerdt, W. B. (2020). The origin of observed magnetic variability for a sol on Mars from InSight. J. Geophys. Res.: Planets, 125(9), e2020JE006505. https://doi.org/10.1029/2020JE006505

Nielsen, E., Zou, H., Gurnett, D. A., Kirchner, D. L., Morgan, D. D., Huff, R., Orosei, R., Safaeinili, A., Plaut, J. J., and Picardi, G. (2006). Observations of vertical reflections from the topside Martian ionosphere. Space Sci. Rev., 126(1-4), 373-388. https://doi.org/10.1007/s11214-006-9113-y

Opgenoorth, H. J., Dhillon, R. S., Rosenqvist, L., Lester, M., Edberg, N. J. T., Milan, S. E., Withers, P., and Brain, D. (2010). Day-side ionospheric conductivities at Mars. Planet. Space Sci., 58(10), 1139-1151. https://doi.org/10.1016/j.pss.2010.04.004

Ramstad, R., Brain, D., Dong, Y., Espley, J., Halekas, J., and Jakosky, B. (2020). The global current systems of the Martian induced magnetosphere. Nat. Astron., 4, 979-985. https://doi.org/10.1038/s41550-020-1099-y 
Schunk, R., and Nagy, A. (2009). lonospheres: Physics, Plasma Physics, and Chemistry (2nd ed). New York: Cambridge University Press. https://doi.org/10.1017/CBO9780511635342

Smrekar, S. E., Lognonné, P., Spohn, T., Banerdt, W. B., Breuer, D., Christensen, U., Dehant, V., Drilleau, M., Folkner, W., ... Wieczorek, M. (2019). Pre-mission InSights on the Interior of Mars. Space Sci. Rev., 215, 3. https://doi.org/10.1007/s11214-018-0563-9

Venkateswara Rao, N., Balan, N., and Patra, A. K. (2014). Solar rotation effects on the Martian ionosphere. J. Geophys. Res.: Space Phys., 119(8), 6612-6622. https://doi.org/10.1002/2014JA019894

Verhoeven, O., and Vacher, P. (2016). Laboratory-based electrical conductivity at Martian mantle conditions. Planet. Space Sci., 134, 29-35. https://doi.org/10.1016/j.pss.2016.10.005

Withers, P., and Mendillo, M. (2005). Response of peak electron densities in the Martian ionosphere to day-to-day changes in solar flux due to solar rotation. Planet. Space Sci., 53(14-15), 1401-1418.

https://doi.org/10.1016/j.pss.2005.07.010
Withers, P., Fallows, K., and Matta, M. (2014). Predictions of electron temperatures in the Mars ionosphere and their effects on electron densities. Geophys. Res. Lett., 41(8), 2681-2686. https://doi.org/10.1002/2014GL059683

Withers, P., Morgan, D. D., and Gurnett, D. A. (2015). Variations in peak electron densities in the ionosphere of Mars over a full solar cycle. Icarus, 251, 5-11. https://doi.org/10.1016/j.icarus.2014.08.008

Xu, W. Y. (1992). Effects of the magnetospheric currents on the $S_{q}$-field and a new magnetic index characterizing $S_{q}$ Dynamo current intensity. J. Geomag. Geoelectr., 44(6), 449-458. https://doi.org/10.5636/jgg.44.449

Xu, W. Y., and Kamide, Y. (2004). Decomposition of daily geomagnetic variations by using method of natural orthogonal component. J. Geophys. Res.: Space Phys., 109(A5), A05218. https://doi.org/10.1029/2003JA010216

Yao, M. J., Cui, J., Wu, X. S., Huang, Y. Y., and Wang, W. R. (2019). Variability of the Martian ionosphere from the MAVEN Radio Occultation Science Experiment. Earth Planet. Phys., 3(4), 283-289.

https://doi.org/10.26464/epp2019029 